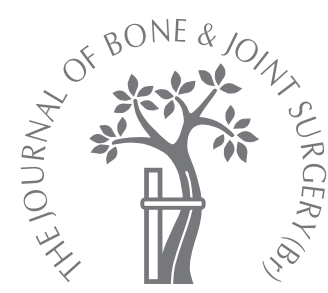

W. W. Duncan, M. J. W. Hubble, J. R. Howell, S. L. Whitehouse, A. J. Timperley, G. A. Gie

From Princess

Elizabeth

Orthopaedic Centre, Royal Devon \& Exeter Hospital, Exeter, England

W. W. Duncan, FRACS, Orthopaedic Surgeon Wakefield Orthopaedic Clinic, 2nd Floor, 270 Wakefield Street Adelaide, South Australia, Australia.

M. J. W. Hubble, FRCS(Tr \& Orth), Consultant Orthopaedic Surgeon

J. R. Howell, FRCS(Tr \& Orth), Consultant Orthopaedic Surgeon

A. J. Timperley, DPhil(Oxon), Consultant Orthopaedic Surgeon

G. A. Gie, FRCSEd(Orth) Consultant Orthopaedic Surgeon

Exeter Hip Unit

Princess Elizabeth Orthopaedic Centre, Royal Devon \& Exeter Hospital, Barrack Road, Exeter EX2 5DW, UK.

S. L. Whitehouse, PhD Research Fellow/Biostatistician Orthopaedic Research Unit, Institute of Health and Biomedical Innovation Queensland University of Technology, The Prince Charles Hospital, Brisbane, Queensland, Australia.

Correspondence should be sent to Mr M. J. W. Hubble; e-mail: matthewhubble@aol.com

(C)2009 British Editorial Society of Bone and Joint Surgery doi:10.1302/0301-620X.91B5. $21621 \$ 2.00$

$J$ Bone Joint Surg $[\mathrm{Br}]$ 2009;91-B:577-82. Received 1 August 2008; Accepted after revision 6 February 2009

\title{
Revision of the cemented femoral stem using a cement-in-cement technique
}

\author{
A FIVE- TO 15-YEAR REVIEW
}

The removal of well-fixed bone cement from the femoral canal during revision of a total hip replacement (THR) can be difficult and risks the loss of excessive bone stock and perforation or fracture of the femoral shaft. Retaining the cement mantle is attractive, yet the technique of cement-in-cement revision is not widely practised. We have used this procedure at our hospital since 1989. The stems were removed to gain a better exposure for acetabular revision, to alter version or leg length, or for component incompatibility.

We studied 136 hips in 134 patients and followed them up for a mean of eight years (5 to 15). A further revision was required in 35 hips (25.7\%), for acetabular loosening in 26 $(\mathbf{1 9 . 1 \% )}$, sepsis in four, instability in three, femoral fracture in one and stem fracture in one. No femoral stem needed to be re-revised for aseptic loosening.

A cement-in-cement revision of the femoral stem is a reliable technique in the medium term. It also reduces the risk of perforation or fracture of the femoral shaft.

The logic behind removing a well-fixed femoral cement mantle at the time of revision total hip replacement (THR) was questioned by Greenwald, Narten and Wilde ${ }^{1}$ over 30 years ago. Instead, they advocated cementing a new component into the old well-fixed cement mantle. When the old cement was prepared by drying and rasping, a shear strength was achieved that was $94 \%$ of that of a single block of cement. Others, however, did not believe that it was possible to achieve a clean dry surface, and in further biomechanical studies found that any contamination with blood or marrow fat reduced the shear strength by up to $85 \% .^{2}$ As a result, or possibly because of the generally poor perception of cemented revision $\mathrm{THR},{ }^{3}$ cement-in-cement femoral revision is not in widespread use.

Despite this, there have been a small number of clinical studies supporting the technique. In 1993, Lieberman et $\mathrm{al}^{4}$ described 19 cases of 'cement-within-cement' femoral revision with no aseptic loosening after a mean of five years, and more recently this has been supported by others. $^{5-7}$

Since 1989, we have routinely performed cement-in-cement femoral revision at our hospital for cases in which the cement mantle appears well fixed clinically and radiologically. Our indications include the removal of the femoral stem to improve access when revising the acetabular component (Fig. 1), to exchange a monobloc stem with a damaged head or a modular stem with a damaged or incompatible taper, to alter the version of the femoral component for instability, to alter leg length for discrepancy, to replace the component for fracture of the stem, or for loosening at the prosthesis cement interface.

The aims of this cohort study were to review the clinical outcome of patients who had undergone revision of a femoral component using this technique and to see how well the implant survived.

\section{Patients and Methods}

Between April 1989 and December 1999 we undertook 191 femoral cement-in-cement revisions in 186 patients. Of these, 122 (66\%) were women and $64(34 \%)$ were men. Their mean age at operation was 70.7 years (29 to 91). A total of 24 patients (25 hips) died before they could be reviewed at five years. All their femoral components remained in place. A further 28 patients (30 hips) did not participate in the five-year review. This group included cement-in-cement revisions for loosening of the acetabular component in 13 , fracture of the stem in one, periprosthetic fracture in one, deep infection in four, dislocation in three, four patients who were in permanent institutional care, and four who had moved abroad. However, all were known to have a functional femoral component with no problems at their last 


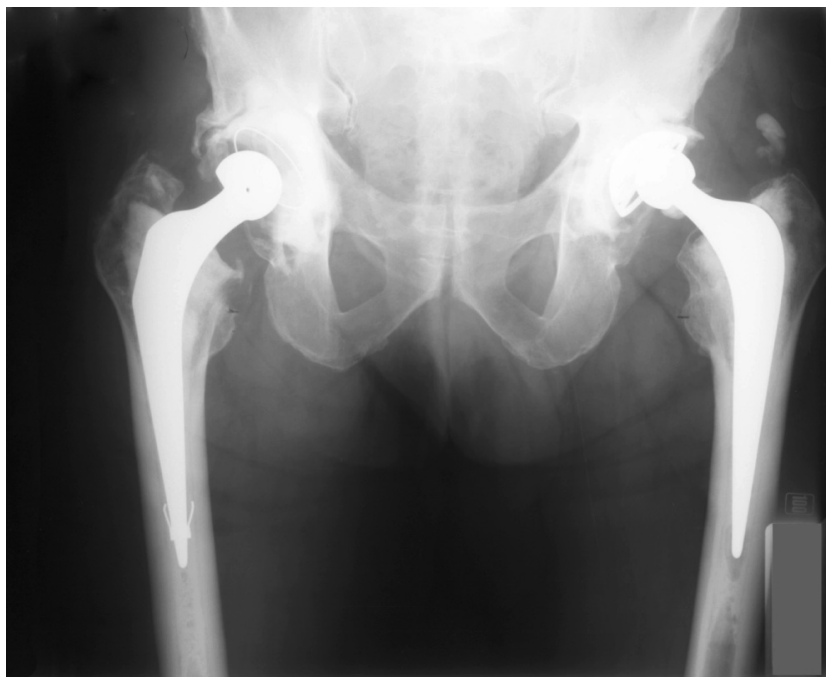

Fig. 1a

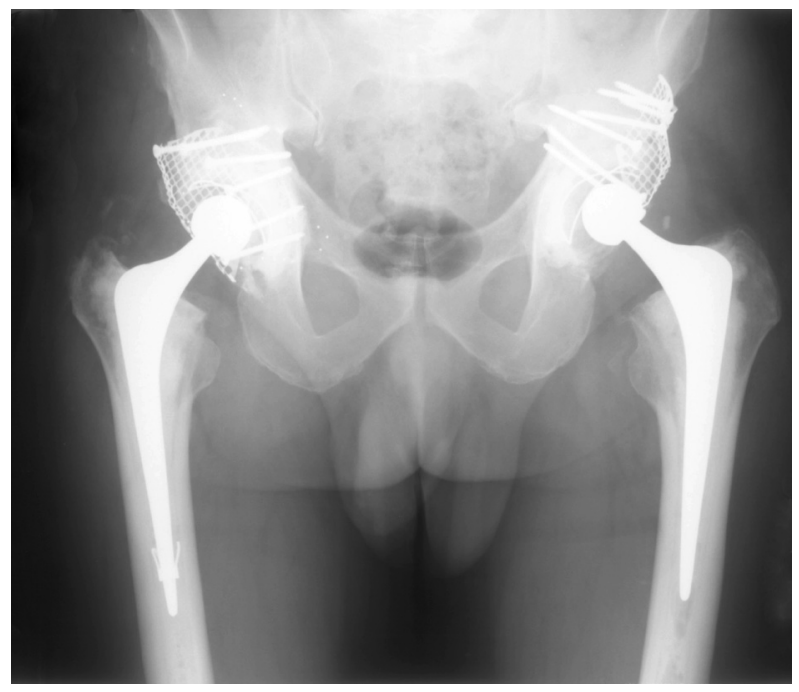

Fig. $1 \mathrm{~b}$

Radiographs showing a) pre- and b) post-operative bilateral acetabular revision with femoral cement-in-cement revision to improve acetabular exposure.

Table I. Median (interquartile range) clinical scores

\begin{tabular}{llll}
\hline Score & Pre-operative & At latest follow-up & p-value $^{*}$ \\
\hline $\begin{array}{l}\text { Harris hip score } \\
\quad \text { Pain (0 to 44, worst to best) }\end{array}$ & $20.0(10)$ & $40.0(24)$ & $<0.001$ \\
$\quad$ Function (0 to 47, worst to best) & $19.5(17)$ & $28.0(17)$ & $<0.001$ \\
& $17.0(13.5)$ & $33.5(16)$ & $<0.001$ \\
Oxford hip score (0 to 48, worst to best) & & $6.0(2)$ & $<0.001$ \\
& & $3.0(3)$ & $<0.001$ \\
Charnley & $3.0(1)$ & $5.0(2)$ & $<.001$ \\
$\quad \begin{array}{l}\text { Pain (0 to 6, worst to best) } \\
\quad \text { Function (0 to 6, worst to best) }\end{array}$ & $2.0(2)$ & \\
$\quad$ Range of movement (0 to 6, worst to best) & $4.0(1)$ & &
\end{tabular}

clinical or radiological review. Therefore, a total of 136 cement-in-cement femoral revisions in 134 patients ( 85 women, 49 men) with complete clinical and radiological follow-up at a minimum of five-years were assessed. No patient was lost to follow-up. The operations were performed by ten different surgeons, all of whom were either consultants or clinical fellows in hip surgery.

The indication for the first revision was acetabular component failure/loosening in 143 of $191(75 \%)$, recurrent dislocation/subluxation in $29(15 \%)$, sepsis in $11(6 \%)$, access for removal of ectopic bone in $4(2 \%)$, and periprosthetic fracture in $4(2 \%)$. In the 136 cases the mean age of the patients at operation was 70.9 years (42 to 91$)$. Of the 136 hips, $86(63 \%)$ were in women and $50(37 \%)$ were in men; $71 \%$ of the initial procedures had been undertaken in Exeter. The mean time from the index operation was 11 years (two months to 25 years). A variety of femoral components were removed, the majority of which were of the Exeter design (Table II). The cement-in-cement revision was the first revision in 102 cases $(75 \%)$, the second in $24(17.6 \%)$, the third in four $(2.9 \%)$, the fourth in one $(0.7 \%)$, and a second stage procedure for infection in five $(3.7 \%)$. Exeter polished stems (Stryker Howmedica, Newbury, United Kingdom) were used in all but three of the cement-in-cement revisions. Of the three original stems which were re-cemented, there was one Charnley (DePuy, Leeds, United Kingdom), one McKeeArden (Deloro Surgical, Swindon, United Kingdom) and one Muller (Zimmer, Warsaw, Indiana).

Clinical evaluation was recorded pre-operatively and at least five years post-operatively using the Harris hip score, ${ }^{8}$ the Oxford hip score, ${ }^{9,10}$ (transformed 0 to 48 worst to best score as recommended by Murray et $\mathrm{al}^{10}$ ) and the Charnley modification $^{11}$ of the d'Aubigné-Postel scoring system (Table I). Statistical analysis was performed by our statistician (SLW) using SPSS version 14.0 for Windows (SPSS Inc., Chicago, Illinois) using Wilcoxon's signed ranks test for non-parametric paired data (as well as marginal homogeneity tests) and presented as median and interquartile range (IQR). The level of significance was $5 \%$, which was adjusted for multiple testing using Bonferroni's method. ${ }^{12}$ 
Table II. Implants removed

\begin{tabular}{ll}
\hline Type of implant & Number (\%) \\
\hline Exeter polished & $74(54.4)$ \\
Exeter matt & $34(25)$ \\
Charnley & $16(11.8)$ \\
McKee-Arden & $3(2.2)$ \\
Müller & $3(2.2)$ \\
Ultima & $2(1.5)$ \\
Howse & $1(0.7)$ \\
Furlong & $1(0.7)$ \\
Lubinus & $1(0.7)$ \\
McKee-Farrar & $1(0.7)$ \\
\hline
\end{tabular}

Table III. Frequency (\%) of Barrack cement mantle grades $^{14}$

\begin{tabular}{lcr}
\hline Grade & Pre-operative & \multicolumn{1}{c}{ 5-year } \\
\hline A & $83(61.0)$ & $94(69.1)$ \\
B & $22(16.2)$ & $31(22.8)$ \\
C & $12(8.8)$ & $9(6.6)$ \\
D & $19(14.0)$ & $2(1.5)$ \\
Total & 136 & 136 \\
\hline
\end{tabular}

Radiographs were taken pre-operatively, immediately post-operatively and at the latest review, and were digitised using the Orthochart system (Ortho-graphics Inc, Salt Lake City, Utah). The cement mantle was assessed and graded using Barrack's ${ }^{13}$ classification, and deficiencies in the mantle recorded by Gruen zone. ${ }^{14}$ The width of any femoral radiolucency at the cement-bone interface was recorded in millimetres and its extent described in Gruen zones. ${ }^{14}$ Subsidence of the stem was recorded in millimetres using the technique of Fowler et al. ${ }^{15}$

All 191 revisions were included in the survival analysis. Kaplan-Meier ${ }^{16}$ survival curves and $95 \%$ confidence intervals $(\mathrm{CI})$ were generated with endpoints for revision of the stem for aseptic loosening and revision for failure from all causes including aseptic loosening, fracture, periprosthetic fracture, infection and dislocation.

There were 13 cases (12 patients) in which the stem was revised a second time using the cement-in-cement technique to allow revision of a failed acetabular component. However, as these cases were not revised for failure of the stem, they were treated as censored data at the time of revision. As no cases were lost to follow-up, construction of a worst-case curve as recommended by Bland ${ }^{12}$ was not warranted.

Operative technique. The proximal cement above the shoulder of the prosthesis was removed using a burr or osteotome before extracting the stem with a punch. A collarless, straight polished tapered stem is straightforward to remove and lends itself to this technique. Collared, curved and mattsurfaced stems can also usually be removed from within the cement mantle by removing more of the proximal cement. The femoral neck was re-cut if necessary to allow the cement-bone interface to be inspected. Any small loose frag- ments of cement above the level of the lesser trochanter were removed. If there was soft tissue at the cement-bone interface which extended distally, if the cement mantle was loose below the lesser trochanter, or if there was loss of host bone, then the cement-in-cement technique was abandoned for a more appropriate method of revision.

A trial stem of appropriate length, offset and version was then chosen to fit inside the existing cement mantle. On occasion, the cement mantle needed to be reshaped with a burr proximally or a reamer distally. Once an adequate trial reduction had been achieved and the position of the stem marked, the mantle was prepared by washing and drying. Fresh polymethylmethacrylate bone cement (Simplex, Stryker, Newbury, United Kingdom) was then introduced in a retrograde manner into the old canal using a cement gun with a narrow (revision) nozzle. It was then pressurised and the new stem inserted in the normal manner.

In most cases we used an Exeter stem with the same offset as the stem that had been removed but with a slimmer body. This allowed exchange of the prostheses with minimal removal of the existing mantle and eliminated the need to remove the distal cement plug. When revising the smaller Exeter or shorter non-Exeter stems, the cement mantle was either reamed distally to accommodate a standard stem, or, if appropriate, the CDH Exeter stem. The Exeter $125 \mathrm{~mm}$ $44 \mathrm{~mm}$ offset cement-in-cement revision stem was not introduced until 2006 and was not available during the period of this study.

In cases in which the stem version had to be altered, considerable cement had to be burred away proximally to allow the stem to rotate within the mantle. In some cases, the distal cement mantle was expanded by a few millimetres with a cylindrical reamer to allow rotation of the stem.

\section{Results}

The mean follow-up was eight years (5 to 15 ).

Of the 136 hips (134 patients) with complete five-year data, the clinical outcome scores were significantly improved after cement-in-cement revision (Table I).

Barrack cement mantle grades were $\mathrm{C}$ or $\mathrm{D}$ in $22.8 \%$ (31 hips) pre-operatively and most of these were grade D (19; $14 \%)$, with gross deficiency at the tip of the prosthesis (Gruen zone 4) (Table III). Only $8.1 \%$ (11 hips) of cement mantles were graded $\mathrm{C}$ or D after cement-in-cement revision and most of these $(5 \%)$ were because of a failure to fill the distal canal with cement. However, this was not statistically significant when tested for marginal homogeneity $(\mathrm{p}=0.21)$. In two cases cement perforation occurred, one at the tip of the prosthesis and one in Gruen zone 2.

At the most recent review, no stem was radiologically loose. There were radiolucencies at the cement-bone interface on the pre-operative radiographs of 21 cases $(15.4 \%)$. Of these, 17 were isolated radiolucencies in the region of the calcar (Gruen zone 7) from $1 \mathrm{~mm}$ to complete loss. There were two isolated zone 1 (greater trochanter) radiolucencies and two combined lesions in zones 5 and 7 and 


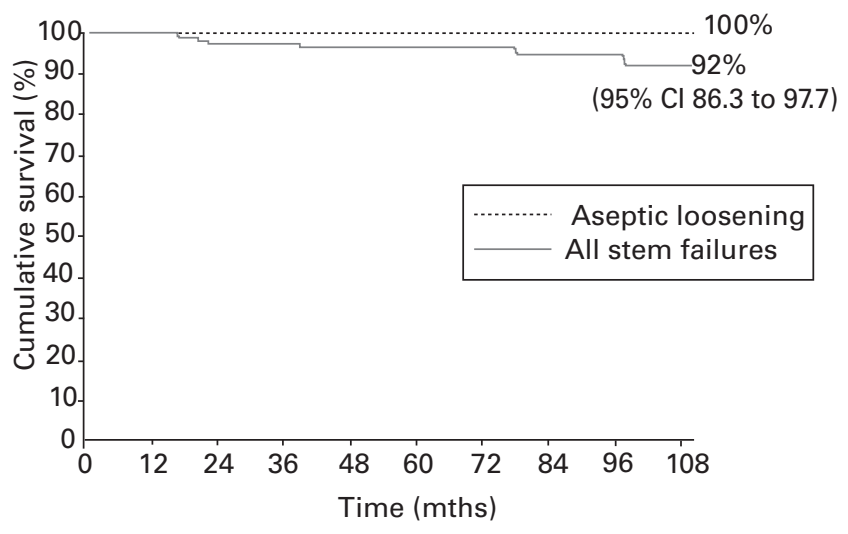

Fig. 2

Cumulative survival curves for aseptic loosening and of the stem revision for any reason (aseptic loosening, fracture, infection and dislocation) $(95 \% \mathrm{Cl}, 95 \%$ confidence interval).

zones 6 and 7. On the initial post-operative radiographs, there was adequate cement fill in seven of the proximal lesions (zones 1 or 7 ). In the 14 cases $(10.3 \%$ ) without adequate cement fill there was no further change in the width or extent of any cement-bone radiolucency on the radiographs taken after five years, other than in one case in which an isolated zone 7 lucency progressed from $2 \mathrm{~mm}$ to complete loss.

In the 104 cases where it was recorded, a new distal centraliser was not used in 59 cases $(57 \%)$. The mean subsidence of 45 of the 104 cases $(43 \%)$ in which a new centraliser was implanted was $0.5 \mathrm{~mm}$ ( 0 to 5$)$ at the latest follow-up, significantly more than those found when no new centraliser was implanted. These subsided a mean of $0.1 \mathrm{~mm}(0$ to 1$)$ ( $\mathrm{p}<0.001)$.

A further revision was carried out in 35 cases $(25.7 \%)$, but none was for aseptic loosening. The mean time to repeat revision was 58.1 months (1 to 156). The indications for repeat revision were failure of the acetabular component in 26 cases $(19.1 \%$ ), sepsis in four, dislocation in three, fracture of the stem in one and periprosthetic fracture in one. A new femoral stem was implanted in 22, and a cement-in-cement technique used for the other 13 .

Survival analysis. There were no failures due to aseptic loosening. The Kaplan-Meier survival for this group was therefore $100 \%$ (95\% CI 91.4 to 100). The survival rate for revision for all causes (aseptic loosening, fracture of the stem, periprosthetic fracture, infection and dislocation) was 92.0\% (95\% CI 86.3 to 97.7) (Fig. 2). We have cited our survival rates at nine years when there were 40 cases remaining at risk. Lettin, Ware and Morris ${ }^{17}$ consider this to be the smallest number of cases that will give a valid survival rate. Yearly survival rates, numbers at risk and 95\% CIs are given in Table IV.

Complications. The femur was perforated in two cases, with escape of cement. One Charnley stem fractured six years after a cement-in-cement revision, having originally been
Table IV. Number remaining at risk and $95 \%$ confidence interval (CI) yearly survival rate, for revision for all reasons

\begin{tabular}{llcl}
\hline Year & Number remaining at risk & \multicolumn{2}{c}{ Survival rate (\%) } \\
\hline 1 & 187 & 100 & \\
2 & 178 & 97.8 & $(95.6$ to 100$)$ \\
3 & 173 & 97.8 & $(95.6$ to 100$)$ \\
4 & 157 & $97.3 \quad(94.9$ to 99.7$)$ \\
5 & 145 & $97.3 \quad(94.9$ to 99.7$)$ \\
6 & 124 & $97.3 \quad(94.9$ to 99.7$)$ \\
7 & 106 & $96.4 \quad(93.5$ to 99.3$)$ \\
8 & 68 & $96.4 \quad(93.5$ to 99.3$)$ \\
9 & 41 & $92.0 \quad(86.3$ to 97.7$)$ \\
\hline
\end{tabular}

implanted for 16 years. At re-revision the cement mantle was found to be well fixed. After removal of the broken stem, a new stem was implanted using the cement-in-cement technique. The cause of the stem fracture is unknown.

Deep sepsis occurred in three cases, two of which required a formal two-stage revision, and the third a Girdlestone procedure. Two cases required revision for dislocation. There was one case of late periprosthetic femoral fracture at 18 months following a fall.

\section{Discussion}

This is the largest series of femoral cement-in-cement revision THRs reported to date: it also has the longest followup. No femoral component has had to be revised for aseptic loosening, although one needed to be revised for fracture of the stem. Survival analysis shows excellent results up to nine years.

Cement-in-cement revision avoids many of the problems associated with the removal of femoral cement. ${ }^{18}$ Removal of well-fixed cement risks bone loss, cortical perforation and fracture, and is time-consuming and technically demanding. To remove sufficient cement to allow the insertion of an uncemented component often requires an extended trochanteric osteotomy or cortical window, which in turn demands long-stemmed distally fixed components. ${ }^{19}$ This extends the 'zone of injury' from the implant further down the femoral canal and increases the difficulty of any subsequent revision procedure. All of these problems can be avoided by using the cement-in-cement technique, making it a quick, versatile and attractive option. Additional advantages are the ability to alter stem offset, version and the depth of insertion, which allow the stem to be adjusted for maximum stability and appropriate leg length at the time of acetabular revision.

Concern has been expressed in the past about the durability of this technique, ${ }^{2}$ but our findings agree with those of others, ${ }^{4-7}$ all of whom have recorded good results with durable fixation and a low failure rate in the short to medium term.

Of the cases that required re-revision of the acetabular component, 13 had a further cement-in-cement stem revision of a well-fixed femoral component to aid exposure at 
the subsequent procedure. A collarless, polished tapered stem lends itself to cement-in-cement revision and effectively makes the stem modular at the stem-cement interface. This means that it can be removed and, if necessary, reimplanted over the lifetime of the replacement.

In many cases the type of cement used was not recorded. Simplex cement was mostly used for both primary and revision procedures in the 96 cases $(71 \%)$ carried out in our unit. In some cases a different cement was used at revision from that used in the primary procedure. This did not appear to have any adverse effect, and nor did the mixing of antibiotic and non-antibiotic cements. These practices have also been supported by in vitro biomechanical research. ${ }^{20}$ As different brands of cement all form a chemical bond with each other to make a solid block of polymethylmethacrylate, it would appear to be acceptable to mix cement types at the time of cement-in-cement revision.

Only one of the 14 radiolucencies that remained after cement-in-cement revision progressed: this was a zone 7 (calcar) defect. Wroblewski ${ }^{21}$ has reported that osteolytic lesions can heal after sealing of defects in the cement mantle, but although the majority of the lesions in our series did not progress after sealing the canal, none became smaller or healed during the course of the study. The radiolucencies that were filled with cement at revision did not recur or develop at other sites.

The current Exeter centraliser (as used in this study) is designed to allow subsidence of the stem so that it engages on the taper-slip principle. ${ }^{22}$ The subsidence seen when components are inserted with distal centralisers has been reported to be the same as that seen after implantation of a primary cemented femoral component. ${ }^{23}$ In those cases in which a new centraliser was not used, the subsidence was significantly less. However, as no stems loosened, and the only stem that fractured was not of a taper-slip design, the significance of this observation, and the need or otherwise for a new centraliser at the time of revision, remains unclear. Our current practice is to use a distal centraliser (usually wingless, and depending on the diameter of the canal within the old cement mantle) at the time of cement-in-cement revision, unless there is a risk of femoral perforation. This ensures that the new stem can function on the taper-slip principle. A specially designed end- and side-cutting reamer with a calibrated shaft (Stryker) is now available which makes the distal reaming required to accommodate the centraliser much easier.

In the case of the Charnley stem which fractured, it must be assumed that the distal part of the stem was well-fixed and the proximal part relatively unsupported. It is imperative that the stem is well supported throughout its length if a cement-in-cement revision is to be performed. In particular, a stem should not be re-cemented into old cement that is well fixed distally but loose proximally. If there is evidence at operation that the proximal cement mantle is loose below the level of the lesser trochanter, we would use an alternative method of revision.
Although it is possible to re-insert an existing stem in its old cement mantle, we prefer not to do so, whatever the design of the old stem, but to insert a new one. This minimises the chance of fatigue failure at a later date and ensures that there is an undamaged trunnion on which to place the new prosthetic femoral head.

Cement-in-cement revision of a cemented stem makes revision easier. Consequently, a collarless, polished tapered stem is much more likely to be removed at revision, whatever the indication. Therefore, there is a risk that a coarse analysis of registry data for stem revision for any reason will show prejudice against stems removed for cement-in-cement revision. The National Joint Registry of England and Wales ${ }^{24}$ includes data sets that recognise both femoral and acetabular cement-incement revision, but this is not universal. It is essential that this technique be correctly registered when comparative data are being analysed if accurate conclusionsare to be drawn.

The authors would like to thank the members of the Exeter Hip Unit, R. Sculpher, S. Wright and Z. Tippett, for their assiduous work in keeping track of every patient, and Mr A. Sheppard who assisted with clinical follow-up.

The author or one or more of the authors have received or will receive benefits for personal or professional use from a commercial party related directly or indirectly to the subject of this article. In addition, benefits have been or will be directed to a research fund, foundation, educational institution, or other nonprofit organisation with which one or more of the authors are associated.

\section{References}

1. Greenwald AS, Narten NC, Wilde AH. Points in the technique of recementing in the revision of an implant arthroplasty. J Bone Joint Surg [Br] 1978;60-B:107-10.

2. Li PL, Ingle PJ, Dowell JK. Cement-within-cement revision hip arthroplasty: should it be done? J Bone Joint Surg [Br] 1996;78-B:809-11.

3. Callaghan JJ, O'Rourke MR, Johnson RC. Revision on the femoral component: cement. In: Callaghan JJ, Rosenberg AG, Rubash HE, eds. The adult hip. Philadelphia:, Lippincott Williams \& Wilkins, 2007:1463-75

4. Lieberman JR, Moeckel BH, Evans BG, Salvati EA, Ranawat CS. Cement-withincement revision hip arthroplasty. J Bone Joint Surg [Br] 1993;75-B:869-71.

5. Nabors ED, Liebelt R, Mattingly DA, Bierbaum BE. Removal and reinsertion of cemented femoral components during acetabular revision. J Arthroplasty 1996;11:146-52.

6. Quinlan JF, O'Shea K, Doyle F, Brady OH. In-cement technique for revision hip arthroplasty. J Bone Joint Surg [Br] 2006;88-B:730-3.

7. Mandziak DG, Howie DW, Neale SD, McGee MA. Cement-within-cement stem exchange using the collarless polished double-taper stem. J Arthroplasty 2007;22:1000-6.

8. Harris WH. Traumatic arthritis of the hip after dislocation and acetabular fractures: treatment by mold arthroplasty: an end-result study using a new method of result evaluation. J Bone Joint Surg [Am] 1969;51-A:737-55.

9. Dawson J, Fitzpatrick R, Carr A, Murray D. Questionnaire on the perceptions of patients about total hip replacement. J Bone Joint Surg [Br] 1996;78-B:185-90.

10. Murray DW, Fitzpatrick R, Rogers $\mathbf{K}$, et al. The use of the Oxford hip and knee scores. J Bone Joint Surg [Br] 2007;89-B:1010-14.

11. Charnley J. Low friction arthroplasty of the hip: theory and practice. Berlin: Springer Verlag, 1979:20-4.

12. Bland JM. An introduction to medical statistics. Second edition. Oxford: Oxford University Press, 1995.

13. Barrack RL, Mulroy RD, Harris WH. Improved cementing techniques and femoral component loosening in young patients with hip arthroplasty: a 12-year radiographic review. J Bone Joint Surg [Br] 1992;74-B:385-9.

14. Gruen TA, McNeice G, Amstutz HC. "Modes of failure" of cemented stem-type femoral components: a radiographic analysis at loosening. Clin Orthop 1979;141:17-27.

15. Fowler JL, Gie GA, Lee AJ, Ling RS. Experience with the Exeter total hip replacement since 1970. Orthop Clin North Am 1988;19:477-89.

16. Kaplan EL, Meier P. Non-parametric estimation from incomplete observations. J Am Stat Assoc 1958;53:457-81.

17. Lettin AW, Ware HS, Morris RW. Survivorship analysis and confidence intervals: an assessment with reference to the Stanmore total knee replacement. J Bone Joint Surg [Br] 1991;73-B:729-31. 
18. Hozack WJ, Wade FA. Removal of components and cement. In: Callaghan JJ, Rosenberg AG, Rubash HE, eds. The adult hip. Philadelphia: Lippincott Williams \& Wilkins, 2007:1352-70

19. Paprosky WG, Martin EL. Removal of well-fixed femoral and acetabular components. Am J Orthop 2002;31:476-8.

20. Weinrauch PC, Bell C, Wilson L, et al. Shear properties of bilaminar polymethylmethacrylate cement mantles in revision hip joint arthroplasty. J Arthroplasty 2007:22:394-403.
21. Wroblewski BM. Wear of the high-density polyethylene socket in total hip arthroplasty and its role in endosteal cavitation. Proc Inst Mech Eng [H] 1997;211:109-18.

22. Shen G. Femoral stem fixation: an engineering interpretation of the long-term outcome of Charnley and Exeter stems. J Bone Joint Surg [Br] 1998;80-B:754-6.

23. Williams HD, Browne G, Gie GA, et al. The Exeter universal cemented femoral component at 8 to 12 years: a study of the first 325 hips. J Bone Joint Surg [Br] 2002;84-B:324-34.

24. No authors listed. The National Joint Registry of England and Wales. www.nrjcentre.org.uk (date last accessed 1 April 2009). 